\title{
Couplage de Ressources d'Interaction
}

\author{
Nicolas Barralon, Christophe Lachenal, Joëlle Coutaz \\ CLIPS-IMAG, Université Jospeh Fourier, 38041, Grenoble Cedex 9, France \\ \{Nicolas.Barralon, Christophe.Lachenal, Joelle.Coutaz\}@imag.fr
}

\begin{abstract}
RESUME
Le couplage de ressources d'interaction est l'un des fondements de l'informatique ambiante où l'utilisateur construit son espace par assemblage d'entités. Dans cet article, nous explorons de manière rationnelle cette notion étudiée jusqu'ici de manière exploratoire. Nous proposons comme point de départ un cycle de vie du couplage dont les états devraient êtres observables et contrôlables de manière souple et robuste par l'utilisateur. Nous illustrons notre propos avec l'état de l'art et les couplages mis en œuvre dans I$\mathrm{AM}$, un intergiciel permettant la construction dynamique d'un espace d'interaction multi-écran, multi-pointeur, multi-clavier.
\end{abstract}

MOTS CLES : Couplage de ressources d'interaction, technique d'interaction, informatique ambiante

\begin{abstract}
Although coupling interaction resources is key to ubiquitous computing, this notion has been overlooked in previous studies. In this paper, we address this notion in a more systematic way by describing coupling as a finite state machine whose states should be maintained observable and controllable by users in a flexible and reliable manner. We illustrate the discussion with state of the art demonstrators as well as with I-AM, a middleware that supports the dynamic construction of multi-screen, multi-keyboard, and multi-pointer interactive spaces.
\end{abstract}

\section{CATEGORIES AND SUBJECT DESCRIPTORS :}

D.2.2 [Software Engineering]: Design Tools and Techniques - /User Interfaces./, H.5.2 [Information Interfaces and Presentation]: User Interfaces.

\section{GENERAL TERMS: Theory, Human Factors}

KEYWORDS : Interaction resources coupling, interaction technique, ubiquitous computing.

\section{INTRODUCTION}

Le domaine de l'informatique subit régulièrement de profonds bouleversements provoqués par l'émergence de nouvelles technologies fondatrices. Aujourd'hui, une nouvelle

Réserver cet espace pour la notice de copyright évolution se prépare avec la convergence des réseaux adhoc sans fil, des micro et nanosystèmes et la maturation de trente années de recherche en systèmes répartis, en perception artificielle et en interaction homme-machine (IHM). Cette convergence de technologies permet d'envisager le passage d'une informatique confinée et statique, à une informatique ambiante favorisant l'émergence opportuniste d'écosystèmes à façon.

Dans cette vision, l'utilisateur passe de sujet à acteur. Dès lors, il jouit de l'usage de dispositifs numériques qu'il peut prêter, emprunter et composer au gré des opportunités. La figure 1 à droite, illustre la situation où deux amis se rencontrant par hasard assemblent leur PDA. Ils obtiennent ainsi une surface d'affichage suffisamment grande pour engager une collaboration. À gauche, le voyageur à proximité d'un mur actif, obtient des renseignements que l'environnement transfère automatiquement au bon format sur le PDA. Ces deux exemples montrent que, par couplage de dispositifs, l'utilisateur obtient de nouveaux services.
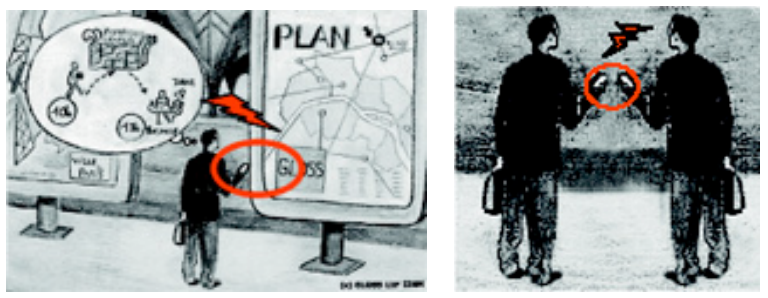

Figure 1. Exemples de couplage opportuniste de ressources d'interaction.

Si le couplage de dispositifs est une dimension importante de l'informatique ambiante, il convient de s'interroger sur sa mise en œuvre technique et son appropriation par l'utilisateur. En particulier, l'utilisateur comprend-il qu'un couplage est possible, peut-il en prédire les effets, sait-il le réaliser, et peut-il en évaluer l'état ? Ces questions nous ont amenés à analyser en détail la notion de couplage de ressources d'interaction avant de réaliser I-AM, un intergiciel permettant la construction dynamique, par l'utilisateur, d'un espace d'interaction multi-surface, multi-pointeur, multi-instrument.

Dans la section qui suit, nous présentons les concepts servant de support à notre raisonnement avec les notions de ressource d'interaction, de surface, d'instrument et de couplage. Puis, nous analysons l'état de l'art aussi bien des IHM conventionnelles pour lesquelles il existe déjà des formes de couplage, que pour les IHM relevant de l'informatique ambiante. Nous constatons que le couplage 
est en état de découverte et que sa maîtrise nécessite une approche rationnelle du problème. Dans ce but, nous proposons comme cadre de réflexion, un cycle de vie du couplage que l'utilisateur doit pouvoir observer et contrôler de manière souple et robuste. Nous illustrons notre propos avec les couplages mis en œuvre dans I-AM.

\section{CONCEPTS : JUSTIFICATION ET DEFINITION}

Les IHM conventionnelles WIMP supposent l'existence de dispositifs d'interaction dont le nombre et le type sont connus par avance : un écran pour les sorties, un clavier et une souris pour les entrées. Avec la réalité mixte (RM), tout objet familier augmenté est un dispositif d'interaction donnant lieu à une grande diversité de types. Avec l'informatique ambiante, à la diversité des types s'ajoute la présence incertaine de dispositifs qui, nous l'avons vu en introduction, peuvent être empruntés, prêtés, composés et découverts dynamiquement. Ces dispositifs, moyens pour construire des espaces interactifs, prennent le statut de ressource. Parce qu'ils sont nécessaires à l'interaction, nous les nommons ressources d'interaction.

La diversité des ressources d'interaction est une source de complexité. En réponse à ce problème, nous avons introduit la notion unificatrice de rôle [3]. Désormais, une entité physique de l'environnement est une ressource d'interaction si elle joue un rôle de surface et/ou d'instrument. Le rôle de surface confère à l'entité la capacité de montrer l'état du système, le rôle d'instrument, celle d'en modifier l'état. Par exemple, un mur peut jouer un rôle de surface et un stylo, celui d'instrument. L'écran d'un PDA sert de surface mais aussi d'instrument lorsque son orientation a un effet sur l'état du système [6].

Pour modéliser la composition dynamique d'un espace interactif, nous utilisons la notion de couplage entre ressources d'interaction. Le couplage de deux ressources d'interaction est l'action de lier ces ressources de manière à ce qu'elles opèrent conjointement pour fournir une nouvelle fonction. Nous illustrons maintenant le couplage de ressources d'interaction dans les IHM conventionnelles avec leurs biens fondés et leurs limitations.

\section{COUPLAGE ET IHM CONVENTIONNELLES}

Dans les IHM conventionnelles, les écrans jouent le rôle de surface tandis que le clavier et les dispositifs de pointage comme la souris, jouent celui d'instrument. Nous allons analyser successivement le couplage d'écrans, le couplage clavier-souris, puis le couplage hybride surface-instrument.

\section{Couplage de surfaces}

Les cartes graphiques des stations de travail permettent la connexion simultanée de plusieurs écrans à une même station de travail. Les systèmes de fenêtrage ont à charge de projeter l'espace logique d'affichage sur la configuration physique d'écrans. Si le système sait détecter la présence des écrans, il est incapable d'en déterminer la configuration spatiale. Ce travail revient à l'utilisateur qui doit spécifier que tel écran est à droite, ou à gauche, de l'écran principal. Le couplage entre écrans est effectif lorsque les deux conditions suivantes sont remplies : connexion physique filaire effectuée, disposition spatiale des écrans spécifiée. En retour, l'utilisateur obtient la fonction "extension de la surface d'affichage". Si l'utilisateur branche un projecteur vidéo, il définit une fonction de couplage en mode miroir, redirection, ou extension.

\section{Couplage clavier-souris}

Dans les IHM WIMP actuelles, l'instrument de pointage et l'instrument de saisie de caractères sont couplés dès lors qu'ils sont connectés au calculateur. La fonction résultant de ce couplage est immuable : l'instrument de pointage détermine le "focus" de l'instrument de saisie. Il lui confère la capacité de multiplexage spatial.

Notons que les touches "flèche" et "tabulation" du clavier permettent aussi de modifier le point d'insertion, mais il s'agit là d'un couplage hybride du clavier avec une surface.

\section{Couplage hybride instrument-surface}

Le couplage entre un instrument de pointage et une surface est effectif dès que l'instrument est physiquement connecté au calculateur et qu'il est déplacé. Dans le cas d'un écran tactile, le couplage a lieu par contact entre la surface et un instrument de forme pointue (qui peut être un doigt).

Le couplage instrument de pointage-surface offre la fonction "désignation d'un point de la surface". Il se traduit par la présence d'un curseur sur la surface dont les déplacements sont asservis aux mouvements de l'instrument de pointage dans les limites de la surface : le curseur ne sort jamais de l'écran confinant les actions de l'utilisateur dans la surface. Notons que si plusieurs instruments de pointage sont connectés à un même ordinateur, un seul, à un instant donné, est couplé à l'écran. Il en va de même pour les écrans tactiles.

Un instrument de saisie de caractères est couplé à l'écran par transitivité de son couplage avec la souris : couplage écran-souris + couplage souris-clavier. Puisque la souris ne peut désigner que des points de l'écran et que la surface d'action du clavier est réduite aux points atteignables par la souris, il est impossible de saisir du texte dans un interacteur hors du bureau. Cette restriction apparente relève d'un bon principe : celui de rendre observables les effets des actions de l'utilisateur.

\section{Synthèse}

En résumé, notre analyse des IHM conventionnelles montre l'existence de couplages dont les effets sont prédéfinis. Certains de ces effets sont directement observables comme l'asservissement du curseur aux mouvements de la souris qui traduit le couplage écran-souris. Inversement, si plusieurs instruments de pointage sont connectés à l'ordinateur, un seul, à un instant donné est couplé à l'écran. Cette application surjective peut, en situation 
d'interaction multi-utilisateur par exemple, paraître quelque peu restrictive. Le couplage clavier-souris est, quant à lui, immuable, et n'est pas directement observable. La configuration des écrans introduit une tâche articulatoire dont la résolution exige de découvrir sur le bureau l'interacteur donnant accès au formulaire de configuration. Enfin, les actions utilisateur, confinées dans l'écran, ne permettent pas d'étendre l'espace d'action au-delà du visible. Qu'en est-il pour les IHM non conventionnelles ?

\section{COUPLAGE ET IHM NON CONVENTIONNELLES}

Une caractéristique que partagent les supports matériels de ces nouvelles IHM est l'absence de fil comme le remarque [5]. Dans ces conditions, le couplage doit s'appuyer sur de nouvelles métaphores. Dans les exemples qui suivent, les gestes synchronisés et la proximité servent systématiquement de fondement à ces métaphores, qu'il s'agisse de couplage de surfaces, d'instruments, ou de couplage hybride.

\section{Couplage de surfaces \\ Gestes synchronisés}

Avec la technique des gestes synchronisés de Hinckley (synchronous gesture), l'utilisateur peut construire dans le plan une mosaïque de surfaces [9]. Chaque surface est une tablette équipée d'accéléromètres qui détectent les collisions de tablettes ainsi que le bord d'impact. Une paire de capteurs de pression placés sur les côtés gauche et droit déterminent si la tablette est tenue.

Le couplage de deux surfaces est effectif si l'utilisateur cogne les surfaces bord à bord. L'état du couplage est rendu observable par un clip sonore métallique et deux flèches qui se font face sur chacune des surfaces pendant 2 secondes. Le découplage a lieu dès que l'une des surfaces est retirée. Le système produit alors un son mat et des flèches brisées apparaissent quelques secondes.

Contrairement aux IHM conventionnelles, la configuration spatiale des surfaces est ici construite par le système à partir des signatures des accéléromètres. Comme pour les IHM conventionnelles, la fonction obtenue est une "extension de surface d'affichage", mais la projection de l'espace logique d'affichage sur la configuration physique dépend de la nature des gestes synchronisés. Si une surface vient heurter une seconde surface immobile (dite surface de base), alors la surface mobile emprunte la base : son espace logique d'affichage s'étale sur la base. Si les surfaces se heurtent de manière symétrique, les espaces d'affichage sont échangés. Comment l'utilisateur peut-il prédire la différence ?

\section{Proximité}

Les tablettes des ConnecTables sont équipées de capteurs et d'antennes RFID [17]. Le couplage a lieu lorsque l'antenne d'une tablette voit le tag de l'autre. Une seule configuration spatiale est autorisée : écrans haut contre haut. La fonction obtenue est une "extension de surface d'affichage" avec fusion des deux espaces logiques sources pour ne former qu'un.
Les Datatiles permettent la construction d'un espace de services par assemblage de tuiles sur un plateau. Une tuile est une petite surface dotée d'un RFID et d'un écran plat à cristaux liquides sur lequel est inscrit le nom du service auquel elle donne accès [14]. Un couplage entre deux tuiles a lieu si ces tuiles sont placées sur le plateau, si les services auxquels elles donnent accès sont compatibles et si l'utilisateur trace au stylo un trait de jointure entre les deux tuiles. Le tracé sert à lever les ambiguïtés car deux tuiles ont pu être placées sur le plateau par inadvertance. Il permet aussi de spécifier le sens des échanges de données entre les tuiles ainsi que le mode, discret ou continu, de ces échanges.

Un retour visuel sur les tuiles traduit l'état du couplage et le mode de communication. La fonction obtenue est une "extension de service" dont la nature dépend du service porté par chacune des tuiles. Par exemple, en couplant une tuile donnant accès à la carte météorologique du jour avec une tuile de navigation dans le temps, on obtient la carte météorologique du jour de son choix. Le découplage a lieu si une tuile est retirée du plateau ou si un trait vertical est tracé à la jointure des deux tuiles.

\section{Couplage d'instruments}

Les interfaces bi-manuelles s'appuient nécessairement sur le couplage d'instruments. Ce couplage est le plus souvent statique. C'est le cas des Toolglass [2], du metaDesk [18], du Holowall [11] comme du système de navigation de Hinckley [8]. Pour ce dernier exemple, un TouchPad et une souris TouchMouse sont couplés de manière permanente. La fonction obtenue est un "pan zoom" graphique.

Avec la Table Magique, le couplage d'instruments est dynamique [1]. Des jetons en plastique, situés sur une table et suivis par un système de vision par ordinateur, permettent de manipuler un espace d'information projeté sur la table. Le couplage de deux jetons a lieu lorsque ces jetons sont mis en contact puis éloignés l'un de l'autre. On assiste ici à des gestes synchronisés qui jouent sur la proximité (contact puis éloignement) d'entités physiques. Le système montre l'existence du couplage par l'affichage d'une boîte élastique asservie aux mouvements des jetons. L'utilisateur a alors à sa disposition la fonction "sélection d'un patch" de la table délimité par la boîte élastique. Le découplage des jetons prend fin lorsque l'un des jetons disparaît (en le retirant de la table ou en le masquant avec la main). Plusieurs couples de jetons peuvent être définis simultanément et fonctionner en parallèle.

\section{COUPLAGE HYBRIDE INSTRUMENTS-SURFACES}

Dans les IHM non conventionnelles, le couplage hybride instruments-surfaces s'appuie sur le couplage préalable de dispositifs électroniques dotés de surfaces et d'instruments.

\section{Gestes synchronisés}

Dans SyncTap, les chocs de Hinckley sont remplacés par l'appui simultané de deux touches dédiées placées chacune 
sur les dispositifs à relier [15]. La fonction qui résulte de ce couplage est une "ouverture de communication ad-hoc" entre deux entités identifiées. À partir de là, le développeur peut définir des couplages de surfaces et d'instruments.

Par exemple, Rekimoto montre comment SyncTap peut remplacer le suivi par vision par ordinateur utilisé dans les Surfaces Augmentées [13] pour détecter la présence de PC portables sur une table augmentée et en déduire la configuration spatiale. Avec la vision artificielle, les relations spatiales entre les écrans de PC portables sont automatiquement calculées, mais exige d'instrumenter l'environnement. Le couplage est représenté par la projection d'un halo sur la table centré sur la position du portable. Avec SyncTap, l'utilisateur doit agir de manière conventionnelle sur la souris d'un PC particulier non mobile (dit maître) : par exemple, en faisant sortir le curseur de la souris du PC-maître par la gauche, il indique la présence d'un portable à sa gauche. Une fois la configuration spatiale des surfaces connue, l'utilisateur dispose de la fonction "extension de surface d'affichage" avec fusion des espaces logiques sources. L'HyperCursor opère un lien visuel lors de la migration d'entités numériques entre les surfaces (fonction dite d'hyperdragging).

\section{Proximité}

En interaction proximale, un couplage de dispositifs a lieu lorsque ces entités, équipées de capteurs RFID et d'infrarouge, sont suffisamment proches et alignées [16]. Ce couplage résulte de l'ouverture d'un canal de communication d'un nouveau type, le nearfield channel, qui assure la sécurité, et l'authentification. Le couplage offre, comme précédemment la fonction de "communication ad-hoc" sur laquelle il est possible de définir de nouvelles fonctions de couplage.

Le couplage de SmartIts s'appuie à la fois sur la proximité et les gestes synchronisés. Le couplage a lieu si deux SmartIts (par exemple un porte-monnaie et une clef de voiture augmentés) sont placés l'un contre l'autre et secoués. La fonction obtenue peut être un "avertissement" dès que les entités couplées s'éloignent trop l'une de l'autre. En l'occurrence, ne pas oublier un porte-monnaie Smart-It lorsque l'on part sans les clefs de la voiture.

\section{Synthèse}

Par couplage opportuniste de ressources d'interaction, l'utilisateur, nous l'avons vu, obtient de nouvelles fonctions. L'idée est séduisante à condition que sa mise en œuvre respecte les principes fondamentaux de l'IHM. Avec les réseaux sans fil, nous perdons la physicalité prégnante des connexions filaires. En compensation, les métaphores auxquelles nos exemples de l'état de l'art ont recours s'appuient sur des notions familières : la proximité et la simultanéité de gestes sur des entités.

Toutefois, on s'est encore peu interrogé sur l'affordance perçue (au sens de Norman [12]) des entités en question. Autrement dit, de quels indices l'utilisateur dispose-t-il pour déterminer a priori que deux entités "vont ensemble" techniquement, sémantiquement et socialement? Et comment peut-il prédire la fonction qu'il obtiendra par une action de couplage ? Pour répondre de manière fondée à ces questions, nous avons étudié et formalisé le cycle de vie d'un couplage de ressources d'interaction.

\section{CyCle de VIE DU COUPLAGE DE RESOURCES D'INTERACTION}

Nous nous plaçons dans le cadre de couplages dynamiques avec l'hypothèse suivante : l'utilisateur doit disposer à tout instant des "indices nécessaires et suffisants" pour juger de l'existence ou de la potentialité de couplage entre deux ressources d'interaction. En réponse à ce questionnement, nous disons que le couplage passe par une suite d'états observables et contrôlables par l'utilisateur. Chaque état se définit au moyen de critères. Nous posons ces critères avant de présenter les conditions de transition entre les états, puis le cycle de vie proprement dit d'un couplage.

\section{Un état : trois critères de vérité}

Soient $\boldsymbol{R}$ l'ensemble des ressources d'interaction, $\boldsymbol{F}$ l'ensemble des fonctions de couplage et $\boldsymbol{C}$ l'ensemble des couplages.

Soient $\mathrm{r} 1$ et $\mathrm{r} 2$ deux ressources d'interaction, $\mathrm{F}$ l'ensemble des fonctions obtenues par le couplage c de r1 avec r2, noté (r1, c, r2) ; avec r1 et r2 $\boldsymbol{R}, \mathrm{F} \subset \boldsymbol{F}$ et $\mathrm{c} \in \boldsymbol{C}$.

Un état de (r1, c, r2), est défini par l'ensemble des trois critères de vérité suivants :

- r1 est (couplée /non couplée) avec r2 pour c,

- r1 est (verrouillée /non verrouillée) pour r2 et pour c,

- r1 est (couplable /non couplable) à r2 pour c, ou bien r1 est (découplable/non découplable) de r2 pour c.

Ou, si l'on utilise les prédicats équivalents :

- Couplée $(r 1, c, r 2)$ est vrai si et seulement si $\mathrm{F} \neq \varnothing$. Si $\mathrm{F}=\varnothing$, Couplée $(\mathrm{r} 1, \mathrm{c}, \mathrm{r} 2)$ est faux et NonCouplée (r1, c, r2) est vrai.

- Verrouillée $(r 1, c, r 2)$ est vrai si l'état de $\mathrm{r} 1$ ne permet pas de changer l'état de $(\mathrm{r} 1, \mathrm{c}, \mathrm{r} 2)$. Ce prédicat traduit l'indisponibilité sociale de r1 pour entrer ou sortir du couplage c avec r2. Par exemple, un utilisateur ne veut pas joindre à l'écran public, l'écran privé de son PDA utilisé à des fins personnelles. L'état de (r1, c, r2) est contraint au statu quo jusqu'à ce que Verrouillée (r1, c, r2) devienne faux ou, de manière duale NonVerrouillée (r1, c, r2) vaut vrai.

- Couplable $(r 1, c, r 2)$ est une expression de prédicats autres que Couplée (r1, c, r2) et Verrouillée (r1, c, r2). Elle regroupe toutes les conditions nécessaires à la réalisation de (r1, c, r2), autres que les conditions sur l'existence de F ou sur le verrouillage de $\mathrm{r} 1$ vis-à-vis de r2 dans c. Par exemple, la compatibilité de forme et de rôle, peuvent être modélisées par Couplable. De manière symétrique, Découplable exprime toutes les conditions, autres que les conditions sur l'existence de F 
ou sur le verrouillage, nécessaires à la réalisation de la destruction de $(\mathrm{r} 1, \mathrm{c}, \mathrm{r} 2)$.

\section{TRANSITIONS}

Les transitions entre états correspondent à des événements dont la prise en compte par le système modifie la valeur de l'un des critères. Ces événements traduisent soit la demande de couplage/découplage (c'est-à-dire la demande de lancement ou de suspension des fonctions de F), soit le verrouillage/déverrouillage de ressource, soit leur couplabilité/découplabilité .

\section{CYCLE DE VIE}

L'automate de la figure 2 modélise le cycle de vie de (r1, c, r2). Un automate similaire modélise celui de (r2, c, r1).

Le cycle de vie comprend deux sous-automates, l'un formé par les états 1, 2, 3, 4 pour lesquels Couplée $(r 1, c, r 2)$ est vrai, l'autre par les états $5,6,7,8$ pour lesquels Couplée $(r 1, c, r 2)$ est faux, les états 4 et 6 servant de passerelle entre les deux sous-automates. L'état 4 correspond à la situation où toutes les conditions sont réunies pour que $(\mathrm{r} 1, \mathrm{c}$, r2) soit réalisé. Il ne manque qu'un événement de demande de couplage pour entrer dans l'état 6 .

À titre d'illustration, déroulons l'automate pour le cas de deux jetons j1 et j2 de la Table Magique. A l'initialisation du système, $(\mathrm{j} 1, \mathrm{c}, \mathrm{j} 2)$ est dans l'état 4 : j1 est ni couplé ni verrouillé et couplable vis-à vis de j2 puisqu'il est sur la table, qu'il est de même couleur, de même forme et de même taille que j2 (le suivi des jetons s'appuie sur un système de vision par ordinateur fondé sur un modèle de couleur). La mise en contact de $\mathrm{j} 1$ et $\mathrm{j} 2$ signifie, pour le système, une demande de couplage. (j1, c, j2) entre dans l'état 6 et la fonction "sélectionner un patch" est disponible. Si l'utilisateur sélectionne un patch, puis masque j2 de la main, (j1, c, j2) revient en 4. Mais comme j1 est maintenant attaché à un patch (couplage instrument-surface), j1 est verrouillé pour $(\mathrm{j} 1, \mathrm{c}, \mathrm{j} 2)$, et $(\mathrm{j} 1, \mathrm{c}, \mathrm{j} 2)$ passe de l'état 4 à l'état 3. (j1, c, j2) reviendra à l'état 4 dès que j1 sera détaché de la surface, c'est-à-dire dès qu'il sera masqué par la main. S'il est verrouillé pour (j1, c, j2), j1 n'est pas verrouillé pour ( $\left.\mathrm{j} 1, \mathrm{c}^{\prime}, \mathrm{j} 2\right)$ où c' donne accès aux fonctions de rotation, de zoom et de destruction de patch. (j1, c', j2) est dans l'état 4. Il passe en 6 dès que l'utilisateur pose j2 à l'intérieur du patch auquel j1 est attaché. L'analyse de ces quelques exemples de couplage montre combien cette opération, simple de principe, soulève de nombreux problèmes potentiels d'utilisabilité. Nous les analysons plus avant dans la section qui suit.

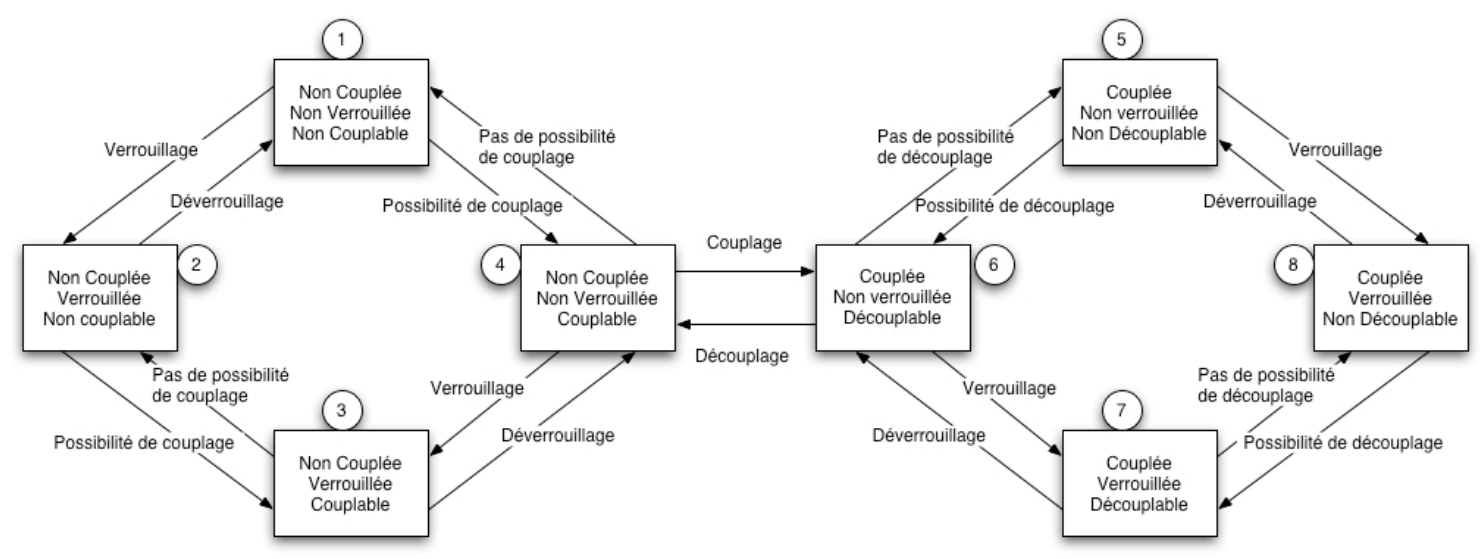

Figure 2. Automate du cycle de vie du couplage ( 1 c c r2). Par souci de clarté du schéma, la fermeture transitive entre états n'est pas représentée, mais les états 1 et 3,2 et 4,5 et 7, 6 et 8 sont évidemment reliés.

\section{COUPLAGE ET PROPRIETES D'INTERACTION}

Nous analysons ci-dessous l'utilisabilité du couplage selon les deux grandes dimensions, souplesse et robustesse de l'interaction introduites dans [7].

\section{Souplesse de l'interaction}

Atteignabilité : "Capacité du système à permettre à l'utilisateur de naviguer dans l'ensemble des états observables du système". Appliquée à notre problème, l'atteignabilité signifie que l'utilisateur doit pouvoir atteindre les états (couplé, verrouillé, couplable) et leur inverse (découplé, déverrouillé, découplable). Il convient donc que l'utilisateur dispose de techniques d'interaction pour atteindre chacun de ces états. Par exemple, pour le verrouillage,
Hinckley [9] propose de masquer avec la main la tablette dont on ne veut pas partager le contenu.

Non-préemption : "Le prochain but souhaité par l'utilisateur est directement atteignable". Pour notre problème, on retiendra que les états souhaités par l'utilisateur doivent être accessibles à tout instant et ceci par des trajectoires d'interaction optimisées. C'est le cas des ConnecTables [17] qui peuvent toujours être couplées ou découplées en un seul geste.

Interaction multifilaire : "Capacité du système à permettre la réalisation de plusieurs tâches". Dans le contexte de notre étude, cette propriété traduit la capacité du système, comme la Table Magique, à traiter plusieurs couplages si- 
multanément ; ou bien, comme cela peut se produire en interaction proximale, plusieurs utilisateurs demandent en même temps le couplage d'entités avec une même entité cible. Soit le système gère l'accès concurrent et l'exprime, soit les conflits sont résolus de manière sociale.

Adaptabilité : "Personnalisation du système sur intervention explicite de l'utilisateur". Aucun exemple de l'état de l'art sur le couplage de ressources d'interaction, n'illustre cette propriété. Couplage/Découplage, Verrouillage/Déverrouillage etc., sont conventionnels et immuables.

Migrabilité de tâche: "Capacité de délégation dynamique de tâches entre le système et l'utilisateur ou entre utilisateurs. C'est un changement dynamique de l'acteur(s) responsable(s) de l'accomplissement de la tâche”. Au regard du couplage, cette propriété s'interprète comme la possibilité de déléguer une partie du couplage au système. Par exemple, les claviers et souris d'une même plateforme sont automatiquement couplés dès le lancement du système.

\section{Robustesse de l'interaction}

Observabilité : "Capacité du système à rendre perceptible l'état pertinent du système". Cette propriété, appliquée à l'automate de couplage, impose de rendre observable l'ensemble des états de l'automate. En particulier, nous avons vu dans l'analyse de l'état de l'art que les états « couplable » et « non couplable » ne sont généralement pas observables.

Honnêteté : "Capacité du système à rendre observable l'état du système sous une forme conforme à cet état et qui engendre une interprétation correcte de la part de l'utilisateur". Autrement dit, l'état du couplage doit non seulement être observable à tout instant, mais ce rendu doit être compris correctement par l'utilisateur. Nous verrons par exemple avec I-AM où l'utilisateur dispose de plusieurs souris et claviers, le problème de représenter le couplage des claviers-souris qui peut varier au cours du temps.

Curabilité : "Capacité pour l'utilisateur de corriger une situation non désirée". Dans l'automate du couplage, cette propriété se traduit par la possibilité de découpler et de déverrouiller à tout instant. La curabilité impose une vigilance toute particulière sur les conséquences du découplage. Par exemple, considérons une surface $\mathrm{S} 1$ couplée à des instruments et à une surface S2. Si l'on découple S1 de S2, doiton pour autant "rapatrier" les instruments de S1 vers la plate-forme de S2, c.-à-d. les recoupler à S2 ou doit-on les perdre et les laisser poursuivre leur vie avec $\mathrm{S} 1$ ?

Prévisibilité : "Capacité pour l'utilisateur de prévoir, pour un état donné, l'effet d'une action". Ici, l'utilisateur doit anticiper les conséquences d'un couplage ou d'un découplage. Nous avons vu chez Hinckley que, selon le geste, le couplage de deux tablettes ne fournit pas le même résultat et que ce résultat, pour un utilisateur néophyte, n'était pas prévisible. Ou encore, prenons l'exemple du couplage de deux souris P1 etP2, et du couplage de deux claviers $\mathrm{C} 1$ et C2. Que se passe-t-il si l'utilisateur couple P1 à C1 ? Le couplage est-il transitif ? Si tel est le cas, P1 devient également couplé à $\mathrm{C} 2$; de même, $\mathrm{P} 2$ est couplé à $\mathrm{C} 1$ et $\mathrm{C} 2$. $\mathrm{Ou}$ encore, si $\mathrm{P} 1$ est couplée à une surface $\mathrm{S} 1$, et $\mathrm{P} 2$ est couplée à une surface S2. Quels sont les effets de bord pour les souris suite au couplage de S1 et S2 ? Pour l'utilisateur, ces conséquences sont-elles prévisibles?

\section{Synthèse}

L'analyse des propriétés de souplesse et de robustesse de l'interaction appliquées à l'automate du couplage de ressources d'interaction, montre, avant toute implémentation, qu'il s'agit d'un problème plus complexe qu'il n'y paraît. Il montre aussi que l'état de l'art s'est peu préoccupé du problème ou s'y est attaché de manière plutôt exploratoire que rationnelle. Il va de soi que la satisfaction de toutes ces propriétés d'utilisabilité n'est pas systématiquement nécessaire. Pour I-AM que nous présentons ci-dessous, nous avons placé la priorité d'une part, sur l'atteignabilité et la non-préemption en faveur de la souplesse d'interaction, d'autre part sur l'observabilité, l'honnêteté, et la curabilité pour la robustesse.

\section{COUPLAGE DE RESSOURCES D'INTERACTION DANS I-AM}

I-AM (Interaction Abstract Machine) se substitue aux gestionnaires de fenêtres conventionnels pour offrir à l'utilisateur, comme au programmeur, un espace d'interaction dynamique multi-écran, multi-clavier, multicurseur, multi-souris comme si ces ressources étaient gérées par un seul ordinateur. La construction de cet espace s'effectue par couplage sous le contrôle de l'utilisateur.

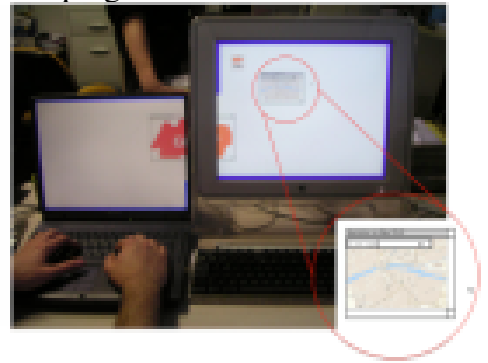

Figure 3. Un espace d'interaction construit avec I-AM à partir des ressources d'interaction d'un PC portable exécutant Windows XP et d'un MacIntosh exécutant MacOSX.

La figure 3 montre l'exemple de deux stations de travail, un PC et un Macintosh, dont les surfaces ont été couplées par les bords droit-gauche. Une fenêtre est affichée à cheval entre les deux écrans. Avec le clavier du PC, l'utilisateur saisit un texte dans l'interacteur affiché sur une surface du Macintosh. Les curseurs des deux machines peuvent migrer sans restriction entre les deux écrans qui forment, grâce à I$\mathrm{AM}$, un seul espace logique d'affichage. On trouvera dans [4] le détail de la mise en œuvre de I-AM. Ici, nous fournissons quelques éléments techniques avant de cibler notre 
analyse sur les techniques de couplage et les problèmes posés sur le plan de l'utilisabilité.

\section{Eléments techniques de I-AM}

Sur chaque ordinateur, s'exécute un PlatformManager responsable de la gestion des ressources d'interaction de cette machine : typiquement, un écran, un clavier, une souris. Il délègue à son SurfaceManager la gestion des surfaces locales, à son InstrumentManager, la gestion de l'ensemble de ses instruments, et aux PhysicalTopologyManager (il y en a un par application s'exécutant sur I-AM), la projection de l'espace logique d'affichage de l'application sur la configuration physique des surfaces. Chaque PhysicalTopologyManager construit son modèle de la configuration physique des surfaces grâce aux publications que chaque PlatformManager émet à propos des caractéristiques des ressources qu'il gère. En particulier, sur chaque bord de surface on peut définir des points de liaison avec les bords d'autres surfaces. Chaque point peut être logiciel ou matérialisé par un capteur d'alignement ou de contact comme dans [9]. Un point de liaison sert de repère pour construire les relations spatiales (supposées planaires) entre surfaces. La mise en correspondance de deux points de liaison de deux surfaces distinctes donne lieu à la création de deux SurfacesLink. Par exemple, un SurfaceLink exprime que la surface $S 1$ voit sur son bord droit en $(x=270 \mathrm{~mm}, y=60$ $\mathrm{mm})$, la surface $\mathrm{S} 2$ par son bord gauche en $(\mathrm{x}=0 \mathrm{~mm}$, $\mathrm{y}=170 \mathrm{~mm}$ ). Dans ce qui suit, nous prendrons l'exemple du $\mathrm{PC}$ et du Macintosh de la figure 3 et étudierons le couplage de surfaces, d'instruments et de surface-instrument. Dans sa version actuelle, I-AM ne gère pas le verrouillage de ressources. Le prédicat Verrouillée ne sera donc pas discuté.

\section{Couplage de surfaces}

Sans I-AM, les deux surfaces ne sont ni couplées, ni couplables (état 1). Lorsque les PlatformManager du PC et du Macinstosh ont publié leurs ressources d'interaction, les deux surfaces deviennent couplables (état 4). Cet état est rendu observable par la présence d'un halo bleu qui entoure chacun des écrans (Figure 4) : chaque halo délimite un espace d'affichage.

Le couplage a lieu par gestes synchronisés façon SynTap, mais en améliorant la prédictibilité de l'effet du couplage. Comme le montre la Figure 4 a), l'utilisateur, une souris dans chaque main, amène les curseurs des souris dans le halo des bords à joindre. En appuyant simultanément sur les boutons des souris, les bords de jointure pâlissent, montrant le passage qui s'ouvrira entre les deux écrans au relâché des boutons. La position du curseur dans le halo désigne de manière directe et dynamique un point de liaison de la surface. En maintenant les boutons enfoncés, jouant sur les positions respectives des points de liaison, l'utilisateur peut ajuster la taille souhaitée de la passerelle et donc la forme du futur espace unifié (Figure 4 b). La passerelle est opérationnelle au relâchement simultané des deux boutons (figure 3). Le halo s'ouvre au niveau de la passerelle pour traduire la continuité de l'espace d'affichage formé par le couplage des deux surfaces.

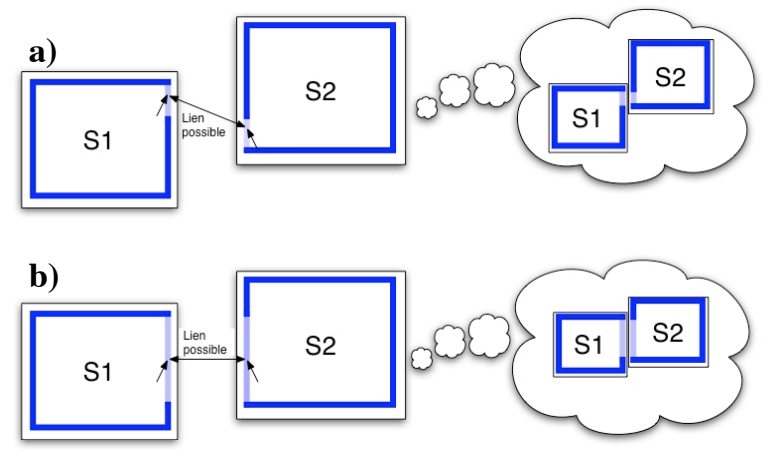

Figure 4. Ajustement dynamique des points de liaison entre surfaces et évaluation a priori de l'espace unifié potentiel.

Pour le découplage, une icône apparaît 2 secondes lorsque le curseur d'une souris passe par la passerelle et que cette souris n'est pas couplée à un interacteur. Un « press » sur l'icône permet de savoir si les surfaces sont découplables et ce que deviendront les frontières (les halos) si le découplage est effectué. Si le «release » a lieu sur l'icône alors que les surfaces sont découplables, le découplage est effectif.

\section{Couplage d'instruments}

Dans sa mise en œuvre actuelle, I-AM couple la souris et le clavier d'un même ordinateur avec la sémantique usuelle. En matière d'utilisabilité, nous ne faisons pas mieux que les IHM conventionnelles : l'état du couplage n'est pas observable.

Ce couplage par défaut entre l'instrument de pointage et l'instrument de saisie de texte d'une même machine peut être surchargé en sorte qu'une souris sans fil puisse aisément fonctionner de concert avec le clavier le plus proche. Nous n'avons pas encore exploité ces possibilités qui, si elles offrent souplesse et puissance, peuvent être source de nombreuses confusions. De même, nous n'avons pas exploré le couplage d'instruments de pointage qui, en fonction des situations, permettrait de fusionner plusieurs curseurs en un seul.

\section{Couplage d'instruments et d'interacteurs}

Le couplage d'un instrument avec un interacteur (ou portion de surface) permet à l'utilisateur de modifier l'état de l'interacteur et donc l'information qu'il représente. Sur ce point, nous n'avons pas été créatifs : la souris définit le focus pour l'instrument de saisie de texte qui lui est couplé. En raison de l'existence de plusieurs dispositifs de pointage, un interacteur de saisie de texte peut être le focus de plusieurs claviers simultanément. Par exemple, dans la Figure 3, l'utilisateur a sélectionné avec la souris du PC l'interacteur de saisie de texte. Il a saisi "I can type" avec le clavier du PC. S'il sélectionne l'interacteur avec la souris du Macintosh, il pourra utiliser l'un et l'autre clavier (comme pour les stations portables avec le clavier intégré et 
un clavier externe). Comme précédemment, I-AM offre une grande souplesse à condition que nous fournissions les moyens à l'utilisateur de comprendre et maîtriser la situation.

\section{CONCLUSIONS}

Dans cet article, nous avons étudié le couplage de ressources d'interaction. Dans les IHM conventionnelles, ce problème est ignoré, puisque les ressources d'interaction sont stables par leurs rôles, leurs types et leur nombre. Il suffit que les utilisateurs apprennent et découvrent les conventions. Cette confortable stabilité ne tient plus si, dans la vision informatique ambiante, l'utilisateur bâtit à façon son espace d'interaction à la faveur des besoins et des ressources disponibles. Dans ces conditions, le couplage devient un problème qu'il convient d'étudier avec attention.

En réponse à ces nouvelles exigences, nous avons proposé un cycle de vie du couplage dont chaque état est une combinaison de trois critères : existence de couplage, verrouillage et couplabilité. À partir de là, il convient de permettre à l'utilisateur de naviguer dans ces états en respectant au mieux les règles de souplesse et de robustesse de l'interaction.

Nous avons illustré l'application de ces règles avec la mise en œuvre d'I-AM. En l'état, le couplage de surfaces respecte la plupart des règles annoncées (atteignabilité, absence de préemption, observabilité, prévisibilité, honnêteté), mais l'adaptabilité des techniques de couplage de surfaces se fait, pour l'instant, par programme seulement et il n'y a pas de migration de tâche (le couplage est toujours explicite sous le contrôle de l'utilisateur).

Nos solutions pour le couplage de surfaces sont inspirées de l'application systématique de règles bien fondées, mais n'ont pas fait l'objet d'évaluations formelles. Quant aux couplages d'instruments et aux couplages d'instruments avec les interacteurs, notre analyse montre la richesse des possibilités. Sur ce point, notre étude ne fait que commencer.

\section{REFERENCES}

1. Bérard, F. The Magic Table: Computer-Vision Based Augmentation of a Whiteboard for Creative Meetings. In IEEE workshop on Projector-Camera Systems, Nice, France, 2003.

2. Bier, E., Stone, M., Pier, K., Buxton, W., DeRose, T. Toolglass and Magic Lenses: The See-Through Interface. In Proc of SIGGRAPH 93, 1993, pp.73-80.

3. Coutaz, J., Lachenal, C., Dupuy-Chessa, S. Ontology for Multi-Surface Interaction. In Proc of Interact'03, Zürich, 2003, pp.447-454.

4. Coutaz, J., Lachenal, C., Barralon, N., Rey, G. Final examples of interaction techniques using multiple interaction surfaces. Delivrable 20, Gloss Project Project Number: IST-2000-26070, 2003.
5. Edwards, K., Grinter, R. At Home with Ubiquitous Computing : seven challenges. In Proc of Ubicomp'01, Atlanta, Georgia, 2001, p.256-272.

6. Harrison, B. L., Fishkin, K. P. ,Gujar, A., Mochon C., Want, R. Squeeze me, Hold me, Tilt Me ! An exploration of Manipulative User Interface. In Proc of ACM CHI'98, Los angeles, 1998, p. 17-24.

7. Gram, Ch., Cockton, G. (Eds.). Design Principles for Interactive Software. Chapman \& Hall, London, 1996.

8. Hinkley, K., Czerwinski, M., Sinclai, M. Interaction and Modeling Techniques for Desktop Two-Handed Input. In Proc of the ACM UIST'98, San Francisco, California, 1998, pp.49-58.

9. Hinckley, K. Synchronous Gestures for Multiple Persons. In Proc of the ACM UIST'03, Vancouver, Canada, 2003, pp.149-158.

10. Holmquist, L.E., Mattern, F., Schiele, B., Alahuhta, P., Beigl M., Gellersen, H.W. Smart-Its Friends: A Technique for Users to Easily Establish Connections between Smart Artefacts. In Ubicomp 2001, Atlanta, Georgia, 2001, pp.116-122.

11. Matsushita, N., Rekimoto, J. Holo Wall: Designing a Finger, Hand, Body, and Object Sensitive Wall. In Proc of the ACM UIST'97, Alberta, Canada, 1997, pp.209210.

12. Norman, D. Affordance, Conventions, and Design. In the May 1999 issue of Interactions, 1999, pp.38-43.

13. Rekimoto, J., Saitoh, M. Augmented Surfaces: A spatially continuous work space for hybrid computing environments. In Proc of ACM CHI'99, Pittsburg, Pennsylvania, 1999, pp.378-385.

14. Rekimoto, J., Ullmer, B., Oba, H. DataTiles: A Modular Platform for Mixed Physical and Graphical Interactions. In Proceedings of ACM CHI'01, Seattle, Washington, 2001, pp.269-276.

15. Rekimoto, J., Ayatsuka, Y., Kohno, M. SyncTap: An Interaction Technique for Mobile Networking. In Proc of MOBILE HCI 2003, Udine, Italy, 2003, pp.104-115.

16. Rekimoto, J., Ayatsuka, Y., Kohno, M., Oba, H. Proximal Interactions: A Direct Manipulation Technique for Wireless Networking. In Proc of Interact'03, Zürich, Switzerland, 2003, pp.511-518.

17. Tandler, P., Prante, T., Müller-Tomfelde, C., Streitz, N., Steinmetz, R. ConnecTables: Dynamic Coupling of Displays for the Flexible Creation of Shared Workspaces. In Proc of the ACM UIST'01, Orlando, Florida, 2001, pp.11-20.

18. Ullmer, B., Ishii, H. The metaDESK: Models and Prototypes for Tangible User Interfaces. In Proc of the ACM UIST'97, Alberta, Canada, 1997, pp.223-232. 